\title{
Information and Communication Complexity of Networked Control Systems
}

\author{
Serdar Yüksel ${ }^{1}$
}

December 30, 2013

\begin{abstract}
Information and communication complexity of a networked control system identifies the minimum amount of information exchange needed between the decision makers (such as encoders, controllers and actuators) to achieve a certain objective, which may be in terms of reaching a target state or achieving a given cost threshold. This formulation does not impose any constraints on the computational requirements to perform the communication or control. Both stochastic and deterministic formulations are considered.
\end{abstract}

Keywords Communication complexity, networked control, information theory

\section{Introduction}

Consider a dynamic team problem with $L$ control stations (these will be referred to as decision makers and denoted by DMs) under the following dynamics and measurement equations

$$
\begin{gathered}
x_{t+1}=f_{t}\left(x_{t}, u_{t}^{1}, \ldots, u_{t}^{L}, w_{t}\right), \quad t=0,1, \cdots \\
y_{t}^{i}=g_{t}^{i}\left(x_{t}, u_{t-1}^{1}, \ldots, u_{t-1}^{L} ; v_{t}^{i}\right),
\end{gathered}
$$

where $i \in\{1,2, \ldots, L\}=: \mathcal{L}$ and $x_{0}, w_{[0, T-1]}, v_{[0, T-1]}^{i}$ are mutually independent random variables with specified probability distributions. Here, we use the notation $w_{[0, t]}:=\left\{w_{s}, 0 \leq s \leq t\right\}$.

The DMs are allowed to exchange limited information, see Figure 1. The information exchange is facilitated by an encoding protocol $\mathcal{E}$ which is a collection of admissible encoding functions described as follows. Let the information available to DM $i$ at time $t$ be:

$$
\mathcal{I}_{t}^{i}=\left\{y_{[1, t]}^{i}, u_{[1, t-1]}^{i}, z_{[0, t]}^{i, j}, z_{[0, t]}^{j, i}, j \in \mathcal{L}\right\},
$$

where $z_{t}^{i, j}$ takes values in $\mathcal{Z}_{t}^{i, j}$ and is the information variable transmitted from DM $i$ to DM $j$ at time $t$ generated with

$$
\mathbf{z}_{t}^{i}=\left\{z_{t}^{i, j}, j \in \mathcal{L}\right\}=\mathcal{E}_{t}^{i}\left(\mathcal{I}_{t-1}^{i}, u_{t-1}^{i}, y_{t}^{i}\right),
$$

and for $t=0, \mathbf{z}_{0}^{i}=\left\{z_{0}^{i, j}, j \in \mathcal{L}\right\}=\mathcal{E}_{0}^{i}\left(y_{0}^{i}\right)$. The control actions are generated with

$$
u_{t}^{i}=\gamma_{t}^{i}\left(\mathcal{I}_{t}^{i}\right),
$$

for all DMs. Define $\log _{2}\left(\left|\mathcal{Z}_{t}^{i, j}\right|\right)$ to be the communication rate from DM $i$ to DM $j$ at time $t$ and $\mathcal{R}\left(\mathbf{z}_{[0, T-1]}\right)=$ $\sum_{t=0}^{T-1} \sum_{i, j \in \mathcal{L}} \log _{2}\left(\left|\mathcal{Z}_{t}^{i, j}\right|\right)$ to be the (total) communication rate. The minimum (total) communication rate over all coding and control policies subject to a design objective is called the communication complexity for this objective.

The above is a fixed-rate formulation for communication complexity, since for any two coder outputs a fixed number of bits is used at any given time. One could also use variable-rate formulations. The variablerate formulation exploits the probabilistic distribution of the system variables, see Cover and Thomas (1991).

\footnotetext{
${ }^{1}$ Department of Mathematics and Statistics, Queen's University, Kingston, Ontario, Canada, K7L 3N6. Research Supported By the Natural Sciences and Engineering Research Council of Canada (NSERC). Email: yuksel@mast.queensu.ca.
} 


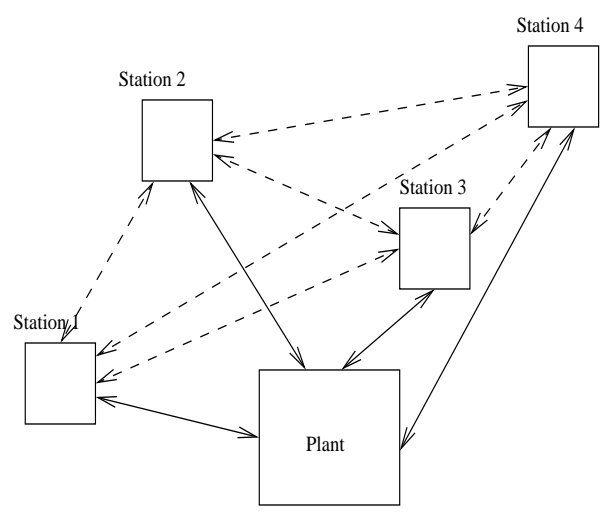

Figure 1: A decentralized networked control system with information exchange between decision makers.

Communication complexity for decentralized dynamic optimization

Let $\underline{\mathcal{E}}^{i}=\left\{\mathcal{E}_{t}^{i}, t \geq 0\right\}$ and $\underline{\gamma}^{i}=\left\{\gamma_{t}^{i}, t \geq 0\right\}$. Under a team-encoding policy $\underline{\mathcal{E}}=\left\{\underline{\mathcal{E}}^{1}, \underline{\mathcal{E}}^{2}, \ldots, \underline{\mathcal{E}}^{L}\right\}$, and a team-control policy $\underline{\gamma}=\left\{\underline{\gamma}^{1}, \underline{\gamma}^{2}, \ldots, \underline{\gamma}^{L}\right\}$, let the induced cost be:

$$
E^{\underline{\gamma}}, \underline{\mathcal{E}}\left[\sum_{t=0}^{T-1} c\left(x_{t}, u_{t}^{1}, u_{t}^{2}, \cdots, u_{t}^{L}\right)\right] .
$$

In networked control, the goal is to minimize (4) over all coding and control policies subject to information constraints in the system. Let $\mathbf{u}_{t}=\left\{u_{t}^{1}, u_{t}^{2}, \cdots, u_{t}^{L}\right\}$. The following definition and example are from Yüksel and Başar (2013).

Definition 0.1 Given a decentralized control problem as above, team cost-rate function $C: \mathbb{R} \rightarrow \mathbb{R}$ is:

$$
C(R):=\inf _{\underline{\gamma}, \underline{\mathcal{E}}}\left\{E \underline{\gamma}, \underline{\mathcal{E}}\left[\sum_{t=0}^{T-1} c\left(x_{t}, \mathbf{u}_{t}\right)\right]: \frac{1}{T} \mathcal{R}\left(\mathbf{z}_{[0, T-1]}\right) \leq R\right\} .
$$

We can define a dual function.

Definition 0.2 Given a decentralized control problem as above, team rate-cost function $R: \mathbb{R} \rightarrow \mathbb{R}$ is:

$$
R(C):=\inf _{\underline{\gamma}, \underline{\mathcal{E}}}\left\{\frac{1}{T} \mathcal{R}\left(\mathbf{z}_{[0, T-1]}\right): E^{\underline{\gamma}}, \underline{\mathcal{E}}\left[\sum_{t=0}^{T-1} c\left(x_{t}, \mathbf{u}_{t}\right)\right] \leq C\right\} .
$$

The formulation here can be adjusted to include sequential (iterative) information exchange given a fixed ordering of actions, as opposed to a simultaneous (parallel) information exchange at any given time $t$. That is, instead of (3), we may have

$$
\mathbf{z}_{t}^{i}=\left\{z_{t}^{i, j}, j \in\{1,2, \ldots, L\}\right\}=\mathcal{E}_{t}^{i}\left(\mathcal{I}_{t-1}^{i}, u_{t-1}^{i}, y_{t}^{i},\left\{z_{t}^{k, i}, k<i\right\}\right) .
$$

Both to make the discussion more explicit and to show that a sequential (iterative) communication protocol may perform strictly better than an optimal parallel communication protocol given a total rate constraint, we state the following example: Consider the following setup with two DMs. Let $x^{1}, x^{2}, p$ be uniformly distributed binary random variables, DM $i$ have access to $y^{i}, i=1,2$, and

$$
x=\left(p, x^{1}, x^{2}\right), \quad y^{1}=p, \quad y^{2}=\left(x^{1}, x^{2}\right),
$$

and the cost function be

$$
c\left(x, u^{1}, u^{2}\right)=1_{\{p=0\}} c\left(x^{1}, u^{1}, u^{2}\right)+1_{\{p=1\}} c\left(x^{2}, u^{1}, u^{2}\right),
$$


with

$$
c\left(s, u^{1}, u^{2}\right)=\left(s-u^{1}\right)^{2}+\left(s-u^{2}\right)^{2} .
$$

Suppose that we wish to compute the minimum expected cost subject to a total rate of 2 bits that can be exchanged. Under a sequential scheme, if we allow DM 1 to encode $y^{1}$ to DM 2 with one bit, then a cost of 0 is achieved since DM 2 knows the relevant information that needs to be transmitted to DM 1, again with one bit: If $p=0, x^{1}$ is the relevant random variable with an optimal policy $u^{1}=u^{2}=x^{1}$, and if $p=1, x^{2}$ is relevant with an optimal policy $u^{1}=u^{2}=x^{2}$, and a cost of 0 is achieved. However, if the information exchange is parallel, then DM 2 does not know which state is the relevant one, and it can be shown that a cost of 0 cannot be achieved under any policy.

The formulation in Definition 0.1 can also be adjusted to allow for multiple rounds of communication per time-stage. Keeping the total rate constant, having multiple rounds can enhance the performance for a class of team problems while keeping the total rate constant.

\section{Communication complexity in decentralized computation}

Yao (1979) initiated the research on communication complexity in distributed computation. This may be viewed as a special case of the setting considered earlier but with finite spaces and in a deterministic and an error-free context: Consider two decision makers (DMs) who have access to local variables $x \in$ $\{0,1\}^{n}, y \in\{0,1\}^{n}$. Given a function $f$ of variables $(x, y)$, what is the maximum (over all input variables $x, y)$ of the minimum amount of information exchange needed for at least one agent to compute the value of the function?: Let $s(x, y)=\left\{m_{1}, m_{2}, \cdots, m_{t}\right\}$ be the communication symbols exchanged on input $(x, y)$ during the execution of a communication protocol. Let $m_{i}$ denote the $i$ th binary message symbol with $\left|m_{i}\right|$ bits. The communication complexity for such a setup is defined as:

$$
R(f)=\min _{\underline{\gamma}, \underline{\mathcal{E}}} \max _{(x, y) \in\{0,1\}^{n} \times\{0,1\}^{n}}|s(x, y)|,
$$

where $|s(x, y)|=\sum_{i=1}^{t}\left|m_{i}\right|$ and $\underline{\mathcal{E}}$ is a protocol which dictates the iterative encoding functions as in (5) and $\underline{\gamma}$ is a decision policy.

For such problems, obtaining good lower bounds is in general challenging. One lower bound for such problems is obtained through the following reasoning: A subset of the form $A \times B$, where $A$ and $B$ are subsets of $\{0,1\}^{n}$ is called an $f$-monochromatic rectangle if for every $x \in A, y \in B, f(x, y)$ is the same. It can be shown that given any finite message sequence $\left\{m_{1}, m_{2}, \cdots, m_{t}\right\}$, the set $\left\{(x, y): s(x, y)=\left\{m_{1}, m_{2}, \cdots, m_{t}\right\}\right\}$ is an $f$-monochromatic rectangle. Hence, to minimize the number of messages, one needs to minimize the number of $f$-monochromatic rectangles which has led to research in this direction. Upper bounds are typically obtained by explicit constructions. For a comprehensive review, see Kushilevitz and Nisan (2006).

For control system, the discussion takes further aspects into account including a design objective, system dynamics and the uncertainty in the system variables.

\section{Communication complexity in reach-control}

Wong (2009) defines the communication complexity in networked control as follows. Consider a design specification where two DMs wish to steer the state of a dynamical system in finite time. This can be viewed as a setting in (1)-(2) with $4 \mathrm{DMs}$, where there is iterative communication between a sensor and a DM, and there is no stochastic noise in the system. Given a set of initial states $x_{0} \in \mathcal{X}_{0}$, and finite sets of objective choices for each DM ( $\mathcal{A}$ for DM $1, \mathcal{B}$ for DM 2), the goal is to ensure that (i) there exists a finite time where both DMs know the final state of the system, (ii) the final state satisfies the choices of the DMs, and (iii) the finite time (when the objective is satisfied) is known by the DMs.

The communication complexity for such a system is defined as the infimum over all protocols of the supremum over the triple of initial states, and choices of the DMs, such that the above is satisfied. That is,

$$
R\left(\mathcal{X}_{0}, \mathcal{A}, \mathcal{B}\right)=\inf _{\underline{\gamma}, \underline{\mathcal{E}}} \sup _{\alpha, \beta, x_{0}} R\left(\underline{\gamma}, \underline{\mathcal{E}}, \alpha, \beta, x_{0}\right),
$$

where $R\left(\underline{\gamma}, \underline{\mathcal{E}}, \alpha, \beta, x_{0}\right)$ denotes the communication rate under the control and coding functions $\underline{\gamma}, \underline{\mathcal{E}}$, which satisfies the objectives given by the choices $\alpha, \beta$ and initial condition $x_{0}$. 
Wong obtains a cut-set type lower bound: Given a fixed initial state, a lower bound is given by $2 D(f)$, where $f$ is a function of the objective choices and $D(f)$ is a variation of $R(f)$ introduced in (6) with the additional property that both DMs know $f$ at the end of the protocol. An upper bound is established by the exchange of the initial states and objective functions also taking into account signaling, that is, the communication through control actions, which is discussed further below in the context of stabilization. Wong and Baillieul (2012) consider a detailed analysis for a real-valued bilinear controlled decentralized system.

\section{Connections with information theory}

Information theory literature has made significant contributions to such problems. An information theoretic setup typically entails settings where an unboundedly large sequence of messages are encoded and functions of which are to be computed. Such a setting is not applicable in a real-time setting but is very useful for obtaining performance bounds (that is, good lower bounds on complexity) which can at certain instances be achievable even in a real-time setting. That is, instead of a single-realization of random variables in the setup of (1)-(2), the average performance for a large number of independent realizations/copies for such problems is typically considered.

In such a context, Definitions 0.1 and 0.2 can be adjusted so that the communication complexity is computed by mutual information Cover and Thomas (1991). Replacing the fixed-rate or variable-rate (entropy) constraint in Definition 0.1 with a mutual information constraint leads to desirable convexity properties for $C(R)$ and $R(C)$. Such an information theoretic formulation can provide useful lower bounds and desirable analytical properties.

We note here the interesting discussion between decentralized computation and communication provided by Orlitsky and Roche (2001), as well as by Witsenhausen (1976) where a probability-free construction is considered and a zero-error (non-asymptotic and error-free) computation is considered in the same spirit as in Yao (1979).

Such decentralized computation problems can be viewed as multi-terminal source coding problems with a cost function aligned with the computation objective. Ma and Ishwar (2011) and Gamal and Kim (2012) provide a comprehensive treatment and review of information exchange requirements for computing. Essential in such constructions is the method of binning, which is a key tool in distributed source coding problems. Binning efficiently designates the enumeration of symbols (which can be confused in the absence of coding) given the relevant information at a receiver DM.

Such problems involve interactive communications as well as multi-terminal coding problems. As mentioned earlier, it is also important to point out that multi-round protocols typically reduce the average rate requirements.

\section{Communication complexity in decentralized stabilization}

An important relevant setting of reach-control is where the target final state is the zero vector: the system is to be stabilized. Consider the following special case of (1)-(2) for an LTI system

$$
x_{t+1}=A x_{t}+\sum_{j=1}^{L} B^{j} u_{t}^{j}, \quad y_{t}^{i}=C^{i} x_{t} t=0,1, \ldots
$$

where $i \in \mathcal{L}$, and it is assumed that the joint system is stabilizable and detectable, but the individual pairs $\left(A, B^{i}\right)$ may not be stabilizable or $\left(A, C^{i}\right)$ may not be detectable. Here, $x_{t} \in \mathbb{R}^{n}$ is the state, $u_{t}^{i} \in \mathbb{R}^{m_{i}}$ is the control applied by station $i$, and $y_{t}^{i} \in \mathbb{R}^{p_{i}}$ is the observation available at station $i$, all at time $t$. The initial state $x_{0}$ is generated according to a probability distribution supported on a compact set $\mathcal{X}_{0} \subset \mathbb{R}^{n}$. We denote controllable and unobservable subspaces at station $i$ by $K^{i}$ and $N^{i}$, and refer to the subspace orthogonal to $N^{i}$ as the observable subspace at the $i$ th station, denoted by $O^{i}$. The information available to station $i$ at time $t$ is $I_{t}^{i}=\left\{y_{[0, t]}^{i}, u_{[0, t-1]}^{i}\right\}$. For such a system (see Figure 2), it is possible for the controllers to communicate through the plant with the process known as signaling which can be used for communication of mode information among the decision makers. Denote by $i \rightarrow j$ the property that DM $i$ can signal to DM $j$. This holds if and only if $C^{j}(A)^{l} B^{i} \neq 0$, for at least one $l, 1 \leq l \leq n$. A directed graph $\mathcal{G}$ among the $L$ stations can be constructed through such a communication relationship.

Suppose that $A$ is such that in its Jordan form, where each Jordan block admits distinct real eigenvalues. Then, a lower bound on the communication complexity (per time-stage) for stabilizability is given by 


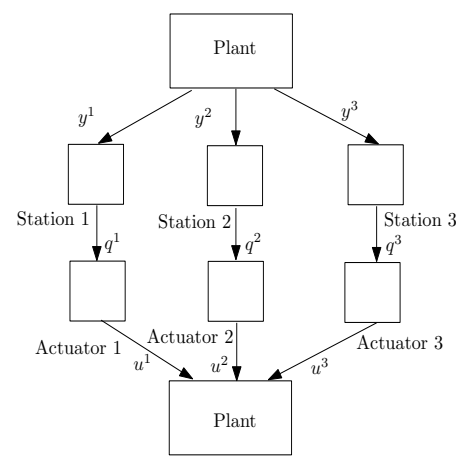

Figure 2: Decentralized stabilization with multiple controllers.

$\sum_{\left|\lambda_{i}\right|>1} \eta_{M_{i}} \log _{2}\left(\left|\lambda_{i}\right|\right)$, where

$$
\eta_{M_{i}}=\min _{l, m \in\{1,2, \ldots, L\}}\left\{d(l, m)+1: l \rightarrow m,\left[x^{i}\right] \subset O^{i} \cup O^{m}, \quad\left[x^{i}\right] \subset K^{m}\right\},
$$

with $d(l, m)$ denoting the graph distance (number of edges in a shortest path) between DM $l$ and DM $m$ in $\mathcal{G}$, and $\left[x_{i}\right]$ denoting the subspace spanned by $x_{i}$. Furthermore, there exist stabilizing coding and control policies whose sum rate is arbitrarily close to this bound. When different Jordan blocks may admit repeated and possibly complex eigenvalues, variations of the result above are applicable. In the special case where there is a centralized controller which receives information from multiple sensors (under stabilizability and joint detectability), even in the presence of noise, to achieve asymptotic stability, it suffices to have the average total rate be greater than $\sum_{\left|\lambda_{i}\right|>1} \log _{2}\left(\left|\lambda_{i}\right|\right)$. The results above follow from Matveev and Savkin (2008) and Yüksel and Başar (2013). For the case with a single sensor, this result has been studied extensively in networked control [see the chapter on Quantized Control and Data-Rate Constraints in the Encyclopedia].

\section{Summary and Future Directions}

In this text, we discussed the problem of communication complexity in networked control systems. Our analysis considered both cost minimization and controllability/reachability problems subject to information constraints. We also discussed the communication complexity in distributed computing as has been studied in the computer science community and provided a brief discussion on the information theoretic approaches for such problems together with structural results. There are many relevant open problems on structural results for optimal policies, explicit solutions as well as non-trivial upper and lower bounds on the optimal performance.

\section{Recommended Reading}

The information exchange requirements for decentralized optimization depend also on the structural properties of the cost functional to be minimized. For a class of team problems, one might simply need to exchange a sufficient statistic needed for optimal solutions. For some problems, there may be no need for an exchange at all, if the sufficient statistics are already available, as in the case of mean field equilibrium problems when the number of decision makers is unbounded or very large for almost optimal solutions; see Huang et al (2006) and Lasry and Lions (2007). In case there is no common probabilistic information, the problem considered becomes further involved. The consensus literature, under both Bayesian and nonBayesian contexts, aims to achieve agreement on a class of system variables under information constraints, see e.g., Tsitsiklis et al (1986). Optimization under local interaction and sparsity constraints and various criteria have been investigated in a number of publications including Rotkowitz and Lall (2006). A review for the literature on norm-optimal control as well as optimal stochastic dynamic teams is provided in Mahajan et al (2012). Tsitsiklis and Athans (1985) have observed that from a computational complexity viewpoint, obtaining optimal solutions for a class of such communication protocol design problems is non-tractable (NP-hard).

Even though obtaining explicit solutions for optimal coding and control results may be difficult, it is useful to obtain structural results on optimal coding and control policies since one can reduce the search space to 
a smaller class of functions. For dynamic team problems, these typically follow from the construction of a controlled Markov chain (see Walrand and Varaiya (1983)) and applying tools from stochastic control theory which obtain structural results on optimal coding and control policies (see Nayyar et al (2013)). Along these lines, for system (1)-(2), if the DMs can agree on a joint beliefs $P\left(x_{t} \in \cdot \mid I_{t}^{i}, i \in \mathcal{L}\right)$ at every time stage, then the optimal cost that would be achieved under a centralized system could be achieved (see Yüksel and Başar (2013)). As a further important illustrative case, if the problem described in Definition 0.1 is for a realtime estimation problem for a Markov source, then the optimal causal fixed-rate coder minimizing any cost function uses only the last source symbol and the information at the controller's memory, see Witsenhausen (1979). We also note that the optimal design of information channels for optimization under information constraints is a non-convex problem; see Yüksel and Linder (2012) and Yüksel and Başar (2013) for a review of the literature and certain topological properties of the problem. We refer the reader to Nemirovsky and Yudin (1983) for a comprehensive resource on information complexity for optimization problems. A sequential setting with an information theoretic approach to the formulation of communication complexity has been considered in Raginsky and Rakhlin (2011). A formulation relevant to the one in Definition 0.1 has been considered in Teneketzis (1979) with mutual information constraints. Giridhar and Kumar (2006) discuss distributed computation for a class of symmetric functions under information constraints and present a comprehensive review.

\section{References}

Cover TM, Thomas JA (1991) Elements of Information Theory. Wiley, New York

Gamal AE, Kim YH (2012) Network Information Theory. Cambridge University Press, U.K.

Giridhar A, Kumar P (2006) Toward a theory of in-network computation in wireless sensor networks. IEEE Communications Magazine 44:98 107

Huang M, Caines PE, Malhamé RP (2006) Large population stochastic dynamic games: closed-loop mckeanvlasov systems and the nash certainty equivalence principle. Communications in Information and Systems 6:221-251

Kushilevitz E, Nisan N (2006) Communication complexity. Cambridge University Press, 2nd ed.

Lasry JM, Lions PL (2007) Mean field games. Japanese J of Mathematics 2:229-260

Ma N, Ishwar P (2011) Some results on distributed source coding for interactive function computation. IEEE Transactions on Information Theory 57:6180-6195

Mahajan A, Martins N, Rotkowitz M, Yüksel S (2012) Information structures in optimal decentralized control. In: IEEE Conference on Decision and Control, Hawaii, USA

Matveev AS, Savkin AV (2008) Estimation and Control over Communication Networks. Birkhäuser, Boston

Nayyar A, Mahajan A, Teneketzis D (2013) The common-information approach to decentralized stochastic control. In: Information and Control in Networks, Editors: G. Como, B. Bernhardsson, A. Rantzer, Springer

Nemirovsky A, Yudin D (1983) Problem Complexity and Method Efficiency in Optimization. WileyInterscience, New York

Orlitsky A, Roche JR (2001) Coding for computing. IEEE Transactions on Information Theory 47:903-917

Raginsky M, Rakhlin A (2011) Information-based complexity, feedback and dynamics in convex programming. IEEE Transactions Inf Theory 57:7036- 7056

Rotkowitz M, Lall S (2006) A characterization of convex problems in decentralized control. IEEE Transactions on Automatic Control 51:274-286

Teneketzis D (1979) Communication in decentralized control. PhD Dissertation, M.I.T. 
Tsitsiklis JN, Athans M (1985) On the complexity of decentralized decision making and detection problems. IEEE Transactions Automatic Contr 30:440-446

Tsitsiklis JN, Bertsekas D, Athans M (1986) Distributed asynchronous deterministic and stochastic gradient optimization algorithms. IEEE Transactions on Automatic Control 31:803-812

Walrand JC, Varaiya P (1983) Optimal causal coding-decoding problems. IEEE Transactions on Information Theory 19:814-820

Witsenhausen HS (1976) The zero-error side information problem and chromatic numbers. IEEE Transactions on Information Theory 22:592-593

Witsenhausen HS (1979) On the structure of real-time source coders. Bell Syst Tech J 58:1437-1451

Wong WS (2009) Control communication complexity of distributed control systems. SIAM J on Control and Optimization 48:1722-1742

Wong WS, Baillieul J (2012) Control communication complexity of distributed actions. IEEE Transactions on Automatic Control 57:2731 2345

Yao ACC (1979) Some complexity questions related to distributive computing. Proceedings of the of the 11th Annual ACM Symposium on Theory of Computing

Yüksel S, Başar T (2013) Stochastic Networked Control Systems: Stabilization and Optimization under Information Constraints. Birkhäuser, Boston, MA

Yüksel S, Linder T (2012) Optimization and convergence of observation channels in stochastic control. SIAM $\mathrm{J}$ on Control and Optimization 50:864-887 